\title{
A Novel Smith Predictive Linear Active Disturbance Rejection Control Strategy for the First-Order Time-Delay Inertial System
}

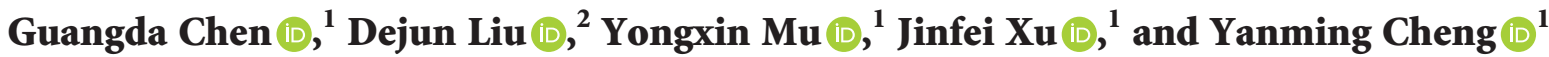 \\ ${ }^{1}$ College of Electrical and Information Engineering, Beihua University, Jilin 132021, China \\ ${ }^{2}$ Engineering Training Center, Beihua University, Jilin 132021, China \\ Correspondence should be addressed to Dejun Liu; liudejun202011@163.com
}

Received 24 February 2021; Revised 2 March 2021; Accepted 4 March 2021; Published 25 March 2021

Academic Editor: Mehmet Emir Koksal

Copyright $\left({ }_{0} 2021\right.$ Guangda Chen et al. This is an open access article distributed under the Creative Commons Attribution License, which permits unrestricted use, distribution, and reproduction in any medium, provided the original work is properly cited.

The control strategy research of the time-delay system is a focused issue in the control field. In order to furthermore improve the performance of the first-order time-delay inertial system, firstly, a new Smith predictor structure is proposed, which solves the constraint that the conventional Smith predictor needs to match the actual object model. Secondly, the performance and parameter function of the new Smith predictor are discussed in theory to provide the basis for parameter tuning. Finally, a new Smith predictor combined with linear active disturbance rejection control (LADRC) is proposed to solve the problem that the two input signals of the linear extended state observer (LESO) are not synchronized on the time scale, and the stability of the new Smith + LADRC time-delay control system is proved theoretically for known and unknown controlled complex objects. Simulation analysis is conducted to verify the robustness of the proposed strategy under the condition of the different parameters. The results indicate that the proposed strategy has better performance than the conventional method in response speed, overshoot, adjustment time, and stability.

\section{Introduction}

Time-delay system widely exists in the industrial production process, which is a difficult problem of process control [1]. Due to the delay of signal, the control quantity cannot be adjusted according to the outputs in time. The longer the time delay is, the more difficult the control is. In addition, the system is time-varying [2]. Improving the control performance of the time-delay system has always been one of the hot spots in the control theory and application fields. The most classical theoretical method is the Smith predictor. However, its prediction ability is greatly affected by disturbance and is sensitive to the variation of parameters of the controlled object. To solve the problem, IBM's Dahlin proposed Dahlin algorithm by designing a suitably digital controller. Although the lag effect is eliminated, there is a ringing effect. When the model error increases, the control quality decreases, and even the system is unstable. Subsequently, many intelligent controls and composite controls that are composed of several kinds of control appeared, such as adaptive control [3,4], model-free control [5], neural networks control [6], predictive control [7], Smith + PID [8], PI + multimodel dynamic matrix control [9], and Fractional Order Proportional-Integral-Derivative [10]. These algorithms are suitable for the case that the object has less time delay or variation of parameters.

Han proposed ADRC (active disturbance rejection control) method, which transforms the design of complex systems into integrated series systems by the observation and compensation of disturbances. For the time-delay system, Han also proposed ignoring the time-delay method for ADRC, increasing order method for ADRC, predictive output method for ADRC, predictive input method for ADRC, delayed input method for ADRC, and other control methods of ADRC for TDS (time-delay systems) [11]. The ignoring time-delay method for ADRC is sensitive to the change of time constant, and the allowable range of timedelay parameters is narrow. The increasing order method for ADRC leads to the increase of controller parameters. Both the predictive output method for ADRC and the predictive 
input method for ADRC require identifying or estimating the pure time-delay parameters. The accuracy of identification or estimation directly affects the control performance. The ADRC sliding mode control method is proposed [12], which adopts a smooth nonlinear function to avoid system vibrating. The ADRC time-delay control method based on inverse decoupling control is proposed [13]. The system model needs to be identified. A practical ADRC control method is proposed for the automatic thickness control system of hot continuous rolling by using linear modeling and parameter optimization [14]. Since the extended state observer (ESO) and the error feedback control law of ADRC contain nonlinear functions, it is difficult to analyze the stability, and many parameters need to be tuned. To solve the problem, Gao used linear functions to process the ESO and error feedback control law and proposed the linear ADRC (LADRC) method $[15,16]$, which makes the stability analysis and parameter tuning easier.

A reduced-order ADRC strategy [17] for the time-delay system is proposed based on the characteristics of the small phase delay of the reduced-order observer. In [18], the stability region parameters of LADRC for time-delay systems are obtained by using the double-track method. Furthermore, the combination of LADRC and prediction is one of the effective methods to solve the large time-delay system. In [19], ADRC generalized predictive control (ADRC-GPC) is proposed, which does not need to identify the exact mathematical model of the object online, and greatly reduces the amount of online calculation. In [20], the stability conditions and parameter perturbation of Smith + LADRC for the first-order large time-delay systems are analyzed. In [21], an improved Smith + LADRC strategy is proposed to nonminimum phase time-delay systems, and the stability and robustness of the system are analyzed. In [22], the robustness of Smith + LADRC under the condition that the model has probably been known is investigated.

Based on the above investigations, a new Smith predictive LADRC method for the first-order time-delay system is proposed, and its prediction mechanism and stability are analyzed under the condition that the controlled object model is known and roughly known through simulations.

\section{Principle of LADRC}

The active disturbance rejection controller is generally composed of three parts: tracking differentiator (TD), state error control law (SECL), and extended state observer (ESO). Its structure is shown in Figure 1. The ESO is the core. $\mathrm{TD}$ is the process of arranging the transition, which mainly affects the overshoot of the system. If the demand is moderate for overshoots, the TD will not be used. When the linear function is used in SECL and ESO, the active disturbance rejection is called linear active disturbance rejection, which is beneficial to the theoretical and engineering design and parameter tuning and easy to use the frequency domain method to analyze the stability in engineering.

The differential equation of the first-order system is expressed as

$$
\dot{y}_{p}=f\left(y_{p}, u, w\right)+b u,
$$

where $y_{p}$ is the system output, $w$ is the external disturbance, $u$ is the system input, $b$ is uncertain control gain, and $f\left(y_{p}, u, w\right)$ is an internal synthesis and external disturbance of the system. If the $d=f\left(y_{p}, u, w\right)+\left(b-b_{0}\right) u$ is observed total disturbance $\left(b_{0}\right.$ is the estimated value of $\left.b\right)$, then the first-order system can be expressed as

$$
\ddot{y}_{p}=d+b_{0} u \text {. }
$$

Suppose $x_{1}=y_{p}, x_{2}=d$, and then equation (2) can be written as the state space expression

$$
\left\{\begin{array}{l}
\dot{x}=A x+B u+E \dot{d} \\
y=C x
\end{array}\right.
$$

where $A=\left[\begin{array}{ll}0 & 1 \\ 0 & 0\end{array}\right], B=\left[\begin{array}{c}b_{0} \\ 0\end{array}\right], C=\left[\begin{array}{ll}1 & 0\end{array}\right], E=\left[\begin{array}{l}0 \\ 1\end{array}\right]$.

Based on equation (3), a linear extended state observer (LESO) is established, and a Luenberger observer for the second-order system is designed as

$$
\left\{\begin{array}{l}
\dot{z}=A z+B u+L\left(y_{p}-\hat{y}\right), \\
\hat{y}=C z
\end{array}\right.
$$

where $z=\left[\begin{array}{ll}z_{1} & z_{2}\end{array}\right]^{T}$ is the state estimation of LESO. $L=$ $\left[\begin{array}{ll}\beta_{1} & \beta_{2}\end{array}\right]^{\mathrm{T}}$ is the observer gain. Control quantity $u$ is written as

$$
u=\frac{k_{p}\left(r-z_{1}\right)-z_{2}}{b_{0}},
$$

where $k_{p}$ is the gain of the proportional controller. For the first-order system, the parameters of LESO and controller are, respectively, [15] as

$$
\begin{aligned}
L & =\left[\begin{array}{ll}
\beta_{1} & \beta_{2}
\end{array}\right]^{T}=\left[\begin{array}{ll}
2 \omega_{0} & \omega_{0}^{2}
\end{array}\right]^{T}, \\
k_{p} & =\omega_{c},
\end{aligned}
$$

where $\omega_{0}$ and $\omega_{c}$ are the bandwidth of LESO and controller, respectively.

\section{Proposed Control Strategy for First-Order Time-Delay System}

A first-order delay system can be represented as

$$
G(s)=\frac{K}{T_{p} s+1} e^{-\tau_{p} s},
$$

where $T_{p}$ is the time constant. $\tau_{p}$ is the delay time, and $K$ is the gain of the object. Its differential equation can be expressed as

$$
\dot{y}_{p}=-\frac{1}{T_{p}} y_{p}+\frac{K}{T_{p}} u\left(t-\tau_{p}\right) .
$$

If $f=-\left(1 / T_{p}\right) y_{p}+\left(K / T_{p}\right) u\left(t-\tau_{p}\right)-b_{0} u$ is a total disturbance, then $\dot{y}_{p}=f\left(y_{p}, u, d\right)+b_{0} u$. It can be 


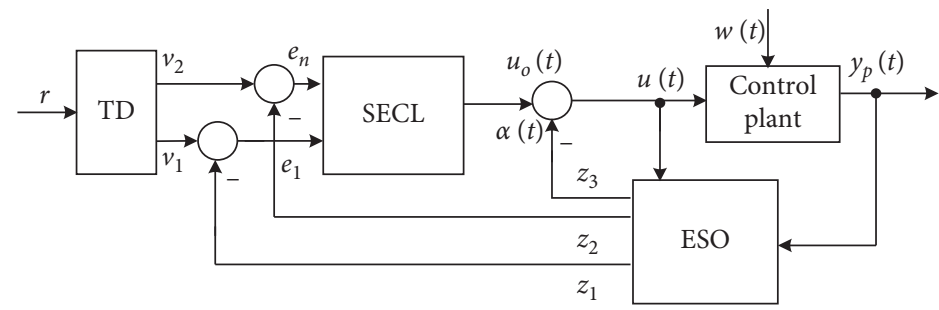

FIgURE 1: Structure diagram of active disturbance rejection controller.

determined from the above equation that inputs $y_{p}\left(t-\tau_{p}\right)$ and $u(t)$ of LESO are not synchronous in time due to the existence of time delay. To estimate the states of the system effectively, two input signals of LESO need to be processed synchronously. It can realize the synchronization of two input signals of LESO by introducing the Smith predictor, but the conventional Smith LADRC will lead to the instability of the system when the parameter perturbation occurs to the object. For this reason, this paper proposes a new method by combining the Smith predictor with linear active disturbance rejection to form a time-delay system controller with strong robustness. Its structure is depicted in Figure 2. Its simplified structure is shown in Figure 3. that

It can be obtained from equations (4) and (6) [20, 21]

$$
\begin{aligned}
& z_{1}=\frac{\omega_{0}^{2}+2 \omega_{0} s}{\left(s+\omega_{0}\right)^{2}} y+\frac{b_{0} s}{\left(s+\omega_{0}\right)^{2}} w, \\
& z_{2}=\frac{\omega_{0}^{2} s}{\left(s+\omega_{0}\right)^{2}} y-\frac{b_{0} \omega_{0}^{2}}{\left(s+\omega_{0}\right)^{2}} w .
\end{aligned}
$$

It can be obtained from the structure diagram in the dashed box in Figure 2 that

$$
\begin{aligned}
& y_{p}(s)=\frac{G_{p} e^{-\tau_{p} s}\left[1+\left(K_{c}+K_{d} s\right) G_{n} e^{-\tau_{n} s}\right]}{\left(1+K_{A} G_{n}\right)\left[1+\left(K_{c}+K_{d} s\right) G_{p} e^{-\tau_{p} s}\right]} \\
& u(s) \quad+\frac{G_{p} e^{-\tau_{p} s}}{1+\left(K_{c}+K_{d} s\right) G_{p} e^{-\tau_{p} s}} w(s) .
\end{aligned}
$$

According to Figure 2 and equation (11), when $y_{p}$ and $y_{n}$ are inconsistent, the feedback controller $K_{c}+K_{d} s$ works, which can restrain the disturbance. $K_{A}$ can make the pole far away from the virtual axis or make the unstable pole become the stable pole [21].

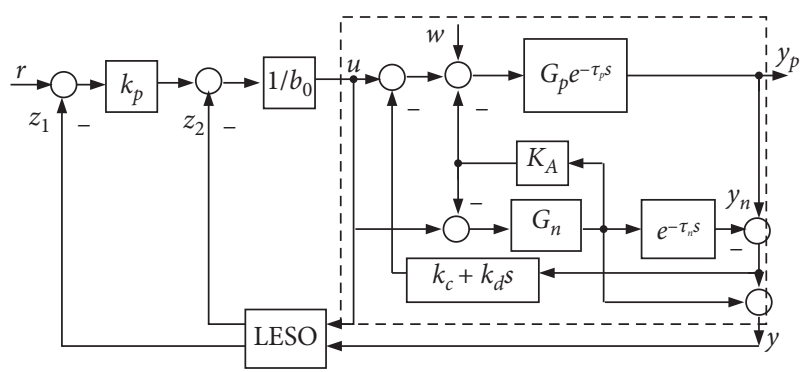

FIgURe 2: Structure diagram of the proposed strategy.

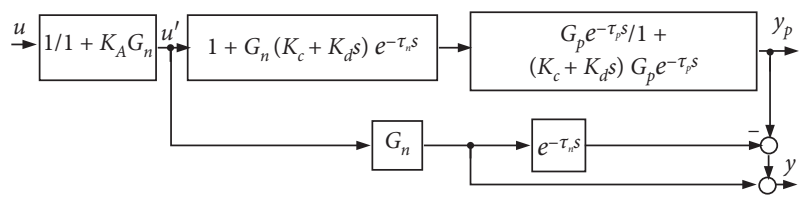

FIGURE 3: Simplified Structure diagram of the proposed strategy.

3.1. The Influence of $K_{c}$ on the Relationship between Disturbances $w$ and $y_{p}$. It can be obtained from equation (11) that

$$
y_{p}(s)=\frac{G_{p} e^{-\tau_{p} s}}{1+\left(K_{c}+K_{d} s\right) G_{p} e^{-\tau_{p} s}} w(s) .
$$

It can be determined from equation (12) that the closedloop characteristic equation is $1+\left(K_{c}+K_{d} s\right) G_{p} e^{-\tau_{p} s}=0$. In order to facilitate the analysis of the time-delay part of the closed-loop characteristic equation, the Pade method is used to approximate $e^{-\tau s}$. The approximation can be express as

$$
e^{-\tau s}=\frac{2-\tau s}{2+\tau s} \text {. }
$$

Then, the closed-loop characteristic equation of $y_{p}(s) / w(s)$ can be written as 


$$
\begin{array}{r}
1+\left(K_{c}+K_{d} s\right) \frac{K}{T_{p} s+1} \frac{2-\tau_{p} s}{2+\tau_{p} s}=0, \\
\left(T_{p} s+1\right)\left(\tau_{p} s+2\right)+\left(K_{c}+K_{d} s\right) K\left(2-\tau_{p} s\right)=0 \\
T_{p} \tau_{p} s^{2}+2 T_{p} s+\tau_{p} s+2+2 K_{c} K-K_{c} K \tau_{p} s+2 K K_{d} s-2 K K_{d} \tau_{p} s^{2}=0 \\
\left(T_{p} \tau_{p}-2 K K_{d} \tau_{p}\right) s^{2}+\left(2 T_{p}+\tau_{p}-K_{c} K \tau_{p}+2 K K_{d}\right) s+2+2 K_{c} K=0 .
\end{array}
$$

If the closed-loop characteristic equation of $y_{p}(s) / w(s)$ is stable, the following conditions must be satisfied:

$$
\begin{array}{r}
T_{p} \tau_{p}-2 K K_{d} \tau_{p}>0, \\
2 T_{p}+\tau_{p}-K_{c} K \tau_{p}+2 K K_{d}>0, \\
2+2 K_{c} K>0 .
\end{array}
$$

$G_{n} e^{-\tau_{n} s}=(2 /(10 s+1)) e^{-10 s}$ is chosen as the object model. The following equation can be obtained:

$$
\begin{array}{r}
T_{p} \tau_{p}-2 K K_{d} \tau_{p}>0 \longrightarrow 10 * 10-2 * 2 * K_{d} * 10>0 \Longrightarrow K_{d}<2.5, \\
2 T_{p}+\tau_{p}-K_{c} K \tau_{p}+2 K K_{d}>0 \longrightarrow 2 * 10+10-K_{c} * 20+2 * 2 * K_{d}>0 \Longrightarrow 15>10 K_{c}-2 K_{d} \\
2+2 K_{c} K>0 \longrightarrow 2+2 * 2 * K_{c}>0 \Longrightarrow K_{c}>-0.5 .
\end{array}
$$

Figure 4 shows the response curve of $y_{p}$ with $K_{A}=2$, $K_{c}=0.3 w=1(t)$ for different $K_{d}$. Figure 5 shows the response curve of $y_{p}$ with $K_{A}=2, K_{d}=0.2 w=1(t)$ for different $K_{c}$.

It can be determined that the overshoot decreases with the increase of $K_{d}$ and the time increases. With the increase of $K_{c}$, the times of adjustment increase and the steady-state value decreases. This indicates that the large $K_{c}$ is beneficial to suppress the disturbance. While the value of $K_{c}$ increases, the adjustment time increases and the response diverges when $K_{c}$ is larger than the critical value.

3.2. The Influence of $K_{A}$ on the Relationship between Control Quantity $u$ and $y_{p}$. When $G_{p}=G_{n}, K_{d}=0.2, K_{c}=0.3$, $\tau_{p}=\tau_{n}$, it can be obtained from equation (10) that

$$
\begin{gathered}
y_{p}(s)=\frac{G_{p} e^{-\tau_{p} s}}{\left(1+K_{A} G_{n}\right)} u(s) \\
=\frac{K e^{-\tau_{p} s}}{T_{p} s+1+K_{A} K} u(s) .
\end{gathered}
$$

When $u(s)=1 / s$, it can be obtained from the final value Theorem 1 that

$$
\begin{array}{r}
e_{s s}(\infty)=\operatorname{lims}_{\substack{s \circledast 0 \\
s \circledast 0}}(s) \\
=s \frac{K e^{-\tau_{p} s}}{T_{p} s+1+K_{A} K} \frac{1}{s}=\frac{K}{1+K_{A} K} .
\end{array}
$$

Figure 6 shows the response curve of $y_{p}$ with $G_{n}=2 /(10 s+1), \tau_{n}=10, u=1(t)$ for different $K_{A}$. It can be determined that, with the increase of $K_{A}$, the more the pole moves to the left, the faster the response is. However, with the increase of $K_{A}$, the output value decreases and the equivalent system gain decreases.

\section{The Analysis of Stability and Robustness}

4.1. The Analysis of Stability. According to Figure 2 and equations (5), (6), (9), (10), and (11), the closed-loop structure diagram is shown in Figure 7.where $G_{m}=\left(G_{n} /\left(1+K_{A} G_{n}\right)\right)\left(1-e^{\tau_{n} s}\right)$. 


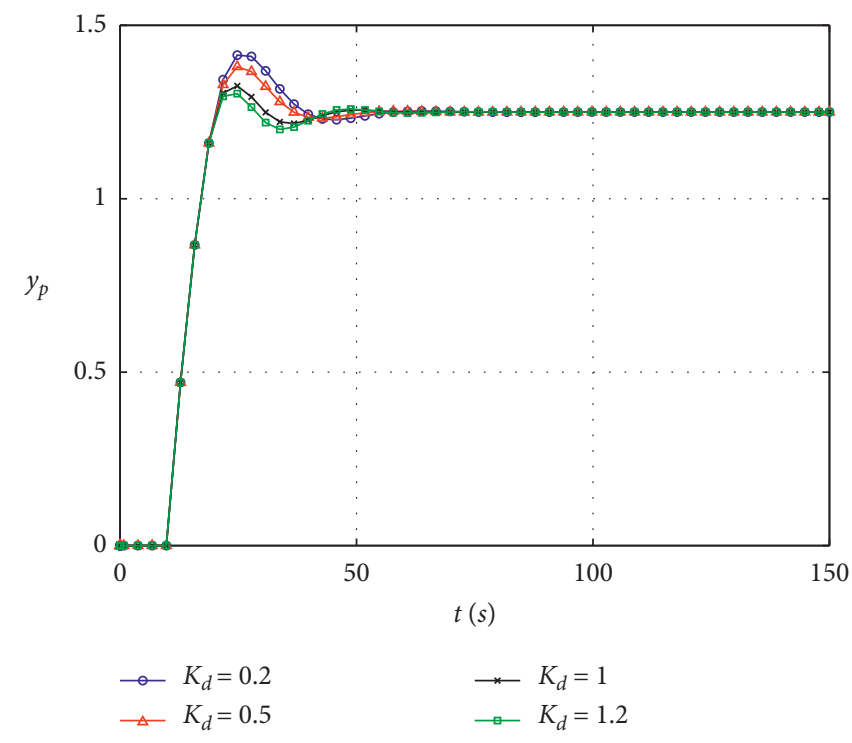

Figure 4: Response curve of $y_{p}$ with $w=1(t)$ for different $K_{d}$.

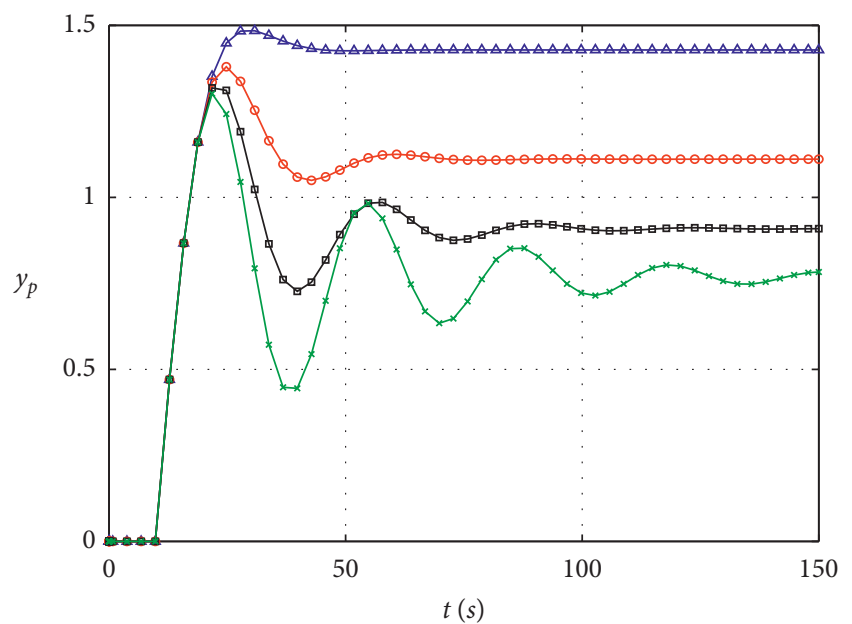

$$
\begin{array}{ll}
\triangle K_{c}=0.2 & \rightarrow K_{c}=0.6 \\
\rightarrow K_{c}=0.4 & \rightarrow-K_{c}=0.8
\end{array}
$$

Figure 5: Response curve of $y_{p}$ with $w=1(t)$ for different $K_{c}$.

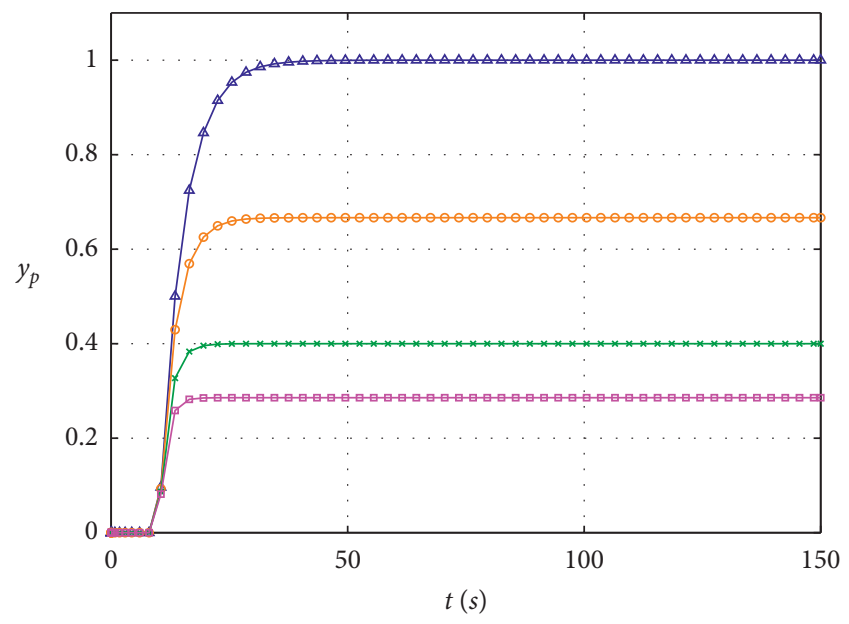

$$
\begin{aligned}
& \triangle K_{A}=0.5 \\
& \therefore K_{A}=1 \\
& \text { * } K_{A}=2 \\
& \rightarrow K_{A}=3
\end{aligned}
$$

Figure 6: Response curve of $y_{p}$ with $u=1(t)$ for different $K_{A}$.

$$
\begin{aligned}
G^{\prime} & =\frac{1}{1+K_{A} G_{n}}\left[1+G_{n} e^{-\tau_{n} s}\left(K_{c}+K_{d} s\right)\right] \frac{G_{p} e^{-\tau_{p} s}}{1+\left(K_{c}+K_{d} s\right) G_{p} e^{-\tau_{p} s}}, \\
H & =\frac{k_{p} \omega_{0}^{2}+2 k_{p} \omega_{0} s+\omega_{0}^{2} s}{k_{p}\left(s+\omega_{0}\right)^{2}}, \\
C & =k_{p}\left(s+\omega_{0}\right)^{2} \cdot\left\{b_{0}\left[s^{2}+\left(2 \omega_{0}+k_{p}\right) s\right]+\left[\left(2 k_{p} \omega_{0}+\omega_{0}^{2}\right) s+k_{p} \omega_{0}^{2}\right] G_{m}\right\}^{-1},
\end{aligned}
$$




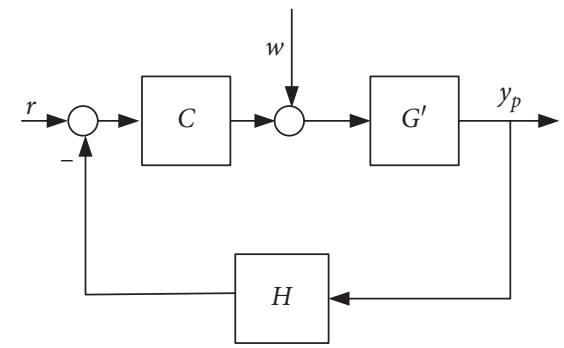

FIGURE 7: The single closed-loop structure diagram of the proposed strategy.

\subsubsection{When the Controlled Object Is Accurately Known}

Theorem 1. When the controlled object is accurately known $\left(K_{A}>0, K_{d}<2.5, K_{c}>-0.5,15>10 K_{c}-2 K_{d}\right)$, and the model parameters of the Smith predictor are consistent with the object parameters, the stable condition of the system depends on $k_{p}, \omega_{0}$ and $b_{0}$.

Prove: According to Figure 7, the closed-loop transfer function is

$$
\begin{aligned}
& G_{c}=\frac{C G^{\prime}}{1+C G^{\prime} H} \\
& =\frac{\left(1 /\left(1+K_{A} G_{n}\right)\right)\left[1+G_{n} e^{-\tau_{n} s}\left(K_{c}+K_{d} s\right)\right]\left(\left(\left(G_{p} e^{-\tau_{p} s} /\left(1+\left(K_{c}+K_{d} s\right) G_{p} e^{-\tau_{p} s}\right)\right) k_{p}\left(s+\omega_{0}\right)^{2}\right) /\left(b_{0}\left[s^{2}+\left(2 \omega_{0}+k_{p}\right) s\right]+\left[\left(2 k_{p} \omega_{0}+\omega_{0}^{2}\right) s+k_{p} \omega_{0}^{2}\right] G_{m}\right)\right)}{1+\left(1 /\left(1+K_{A} G_{n}\right)\right)\left[1+G_{n} e^{-\tau_{n} s}\left(K_{c}+K_{d} s\right)\right]\left(\left(\left(G_{p} e^{-\tau_{p} s} /\left(1+\left(K_{c}+K_{d} s\right) G_{p} e^{-\tau_{p} s}\right)\right) k_{p}\left(s+\omega_{0}\right)^{2}\right) /\left(b_{0}\left[s^{2}+\left(2 \omega_{0}+k_{p}\right) s\right]+\left[\left(2 k_{p} \omega_{0}+\omega_{0}^{2}\right) s+k_{p} \omega_{0}^{2}\right] G_{m}\right)\right) \cdot\left(\left(k_{p} \omega_{0}^{2}+2 k_{p} \omega_{0} s+\omega_{0}^{2} s\right) /\left(k_{p}\left(s+\omega_{0}\right)^{2}\right)\right)} .
\end{aligned}
$$

When the model parameters of the Smith predictor are consistent with the object parameters $\left(K=K_{n}, \tau_{p}=\tau_{n}\right.$, $\left.T_{p}=T_{n}\right)$,

$$
G_{c}=\frac{K_{n} e^{-\tau_{p} s} k_{p}\left(s+\omega_{0}\right)^{2}}{b_{0}\left[s^{2}+\left(2 \omega_{0}+k_{p}\right) s\right]\left[T_{n} s+1\right]+\left[\left(2 k_{p} \omega_{0}+\omega_{0}^{2}\right) s+k_{p} \omega_{0}^{2}\right] K_{n}} .
$$
is

Then, the closed-loop characteristic equation of the system

$$
D(s)=b_{0} T_{n} s^{3}+\left[b_{0}+b_{0} T_{n}\left(2 \omega_{0}+k_{p}\right)\right] s^{2}+b_{0}\left(2 \omega_{0}+k_{p}\right)
$$
$s+K_{n}\left(2 k_{p} \omega_{0}+\omega_{0}^{2}\right) s+K_{n} k_{p} \omega_{0}^{2}$ Let $a_{3}=b_{0} T_{n}, a_{2}=b_{0}+$ $b_{0} T_{n}\left(2 \omega_{0}+k_{p}\right), a_{1}=b_{0}\left(2 \omega_{0}+k_{p}\right)+K_{n}\left(2 k_{p} \omega_{0}+\omega_{0}^{2}\right), a_{0}=$ $K_{n} k_{p} \omega_{0}^{2}$. According to Routh stability criterion, $a_{3}, a_{2}, a_{1}$, $a_{0}>0$ and the system is stable when $a_{1} a_{2}>a_{0} a_{3}$ :

$$
a_{1} a_{2}-a_{0} a_{3}=b_{0}\left\{\left(2 b_{0} \omega_{0}+b_{0} k_{p}+2 K_{n} k_{p} \omega_{0}\right)\left[\left(1+2 T_{n} \omega_{0}\right)+T_{n} k_{p}\right]+K_{n} \omega_{0}^{2}\left(1+2 T_{n} \omega_{0}\right)\right\}>0 .
$$

4.1.2. When Object Model Parameters Are Unknown. Supposing the nominal model of the object is $G_{b}=G_{n} e^{-\tau_{n} s}$, then the actual object is $G(s)=G_{b}(1+\delta G)$. $\delta G$ is the perturbation of the nominal model and stratifies $|\delta G(j \omega)| \leq \overline{\delta G(\omega)} . \overline{\delta G(\omega)}$ is the bounded uncertainty of the multiplicative norm.

From the closed-loop characteristic equation $1+C H G^{\prime}=0$,

$$
1+C H \frac{1}{1+K_{A} G_{n}}\left[1+G_{b}\left(K_{c}+K_{d} s\right)\right] \frac{G_{b}(1+\delta G)}{1+\left(K_{c}+K_{d} s\right) G_{b}(1+\delta G)}=0 .
$$

Set $G_{F}=K c+K_{d} s$. According to the robust stability criterion, for arbitrary $\omega$, the following equation is stratified:

$$
\overline{\delta G(\omega)}<\Delta G(\omega)=\left|\frac{1+K_{A} G_{b}+G_{F}+K_{A} G_{n}+C H+C H G_{b} G_{F} G_{b}}{\left(G_{F}+K_{A} G_{F} G_{n}+C H+C H G_{b} G_{F}\right) G_{b}}\right| .
$$

Therefore, it can be determined that the stability and robustness of the system can be verified by selecting an appropriate value of $b_{0}, k_{p}, K_{A}, K_{c}, K_{d}$, and $\omega_{0}$.
4.2. Robustness Analysis of the Proposed Control Strategy. To analyze the robustness of our proposed control strategy in the dashed box of Figure 2, $G_{n} e^{-\tau_{n} s}=(2 /(10 s+1)) e^{-10 s}$ is supposed as the nominal model of the object and setting $K_{c}=0.5, K_{d}=0.2$, and $K_{A}=2$. The response curve of $y_{p}$ with $u=1(t)$ for different $\tau_{p}$ is shown in Figure 8 . It can be determined from the response curve of simulation that the response will overshoot when $\tau_{p}>\tau_{n}$. The larger the value of $\tau_{p}$ is, the larger the overshoot is, and the longer the adjustment time is. Although the delay time of the system becomes shorter, the rise time increases when $\tau_{p}<\tau_{n}$.

The response curve of $y_{p}$ with $u=1(t)$ for different $T_{p}$ is shown in Figure 9. It can be determined from the response curve of simulation that the overshoot decreases, the rise time increases, and the larger the $T_{p}$ is, the longer the rise time is when $T_{p}>T_{n}$. When $T_{p}<T_{n}$, the rise time decreases, but the overshoot occurs. The longer the adjustment time is, the smaller the $T_{p}$ is and the larger the overshoot is.

The response curve of $y_{p}$ with $u=1(t)$ for different $K$ is shown in Figure 10. It can be determined from the response curve of simulation that the overshoot occurs when $K>K_{n}$. With $K$ becomes larger, the overshoot becomes larger, the 


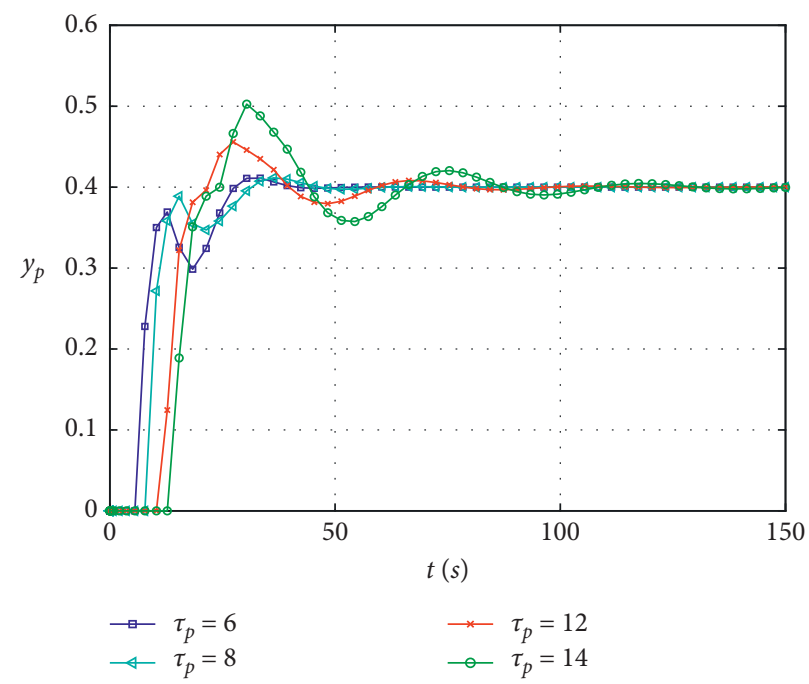

Figure 8: Response curve of $y_{p}$ with $u=1(t)$ for different $\tau_{p}$.

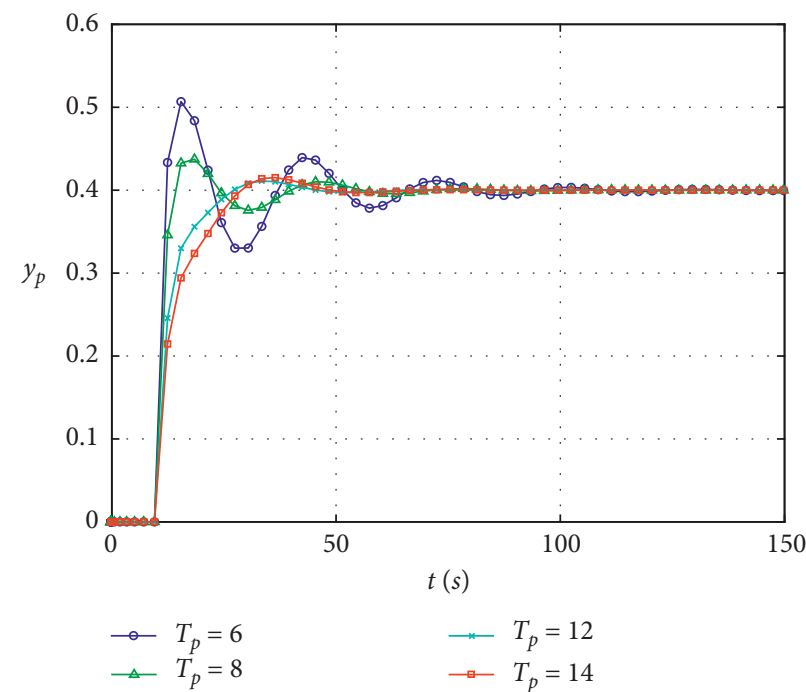

FIgURE 9: Response curve $y_{p}$ with $u=1(t)$ for different $T_{p}$.

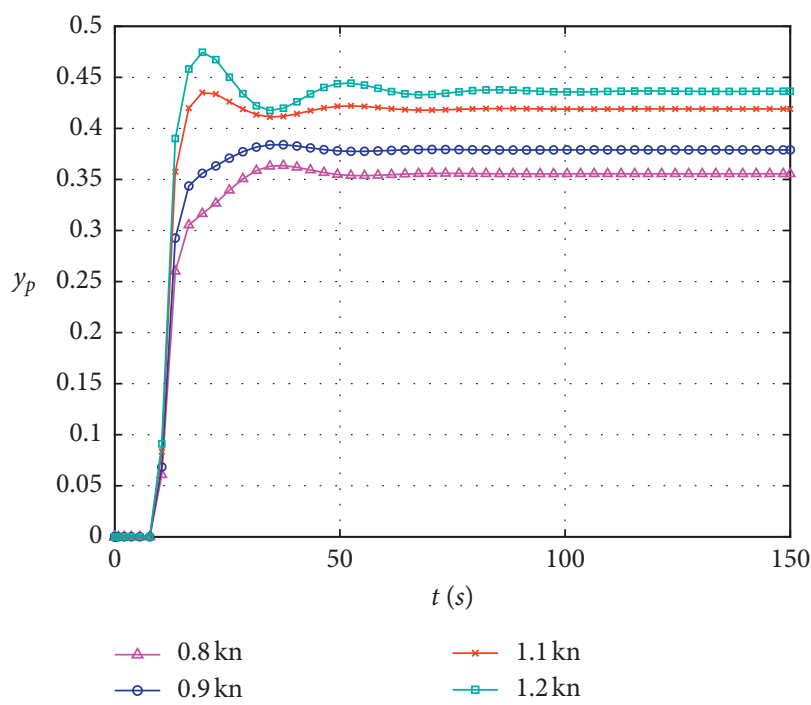

FIGURE 10: Response curve of $y_{p}$ with $u=1(t)$ for different $K$. 


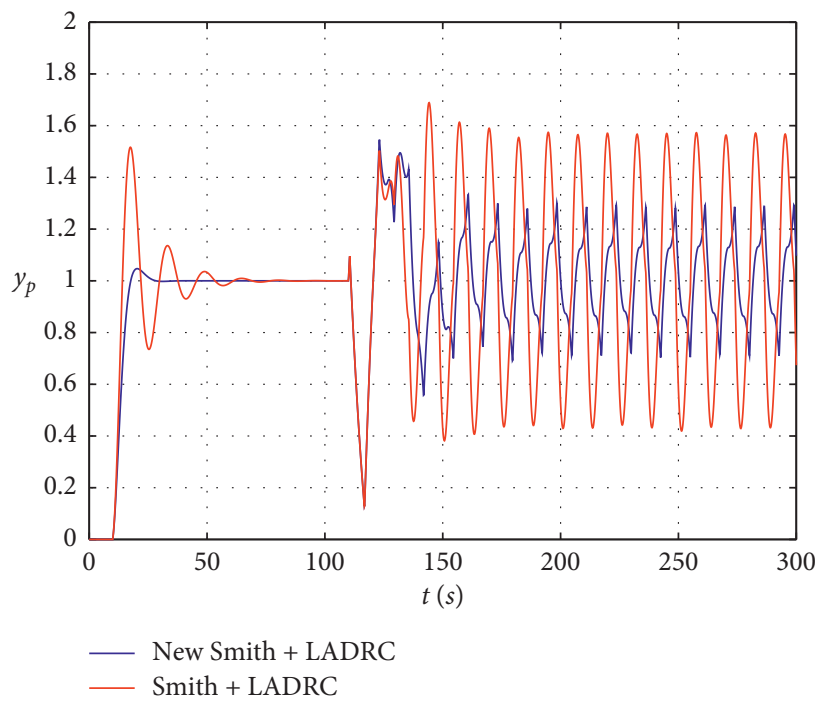

FIgURE 11: Output response curve with square wave disturbance.

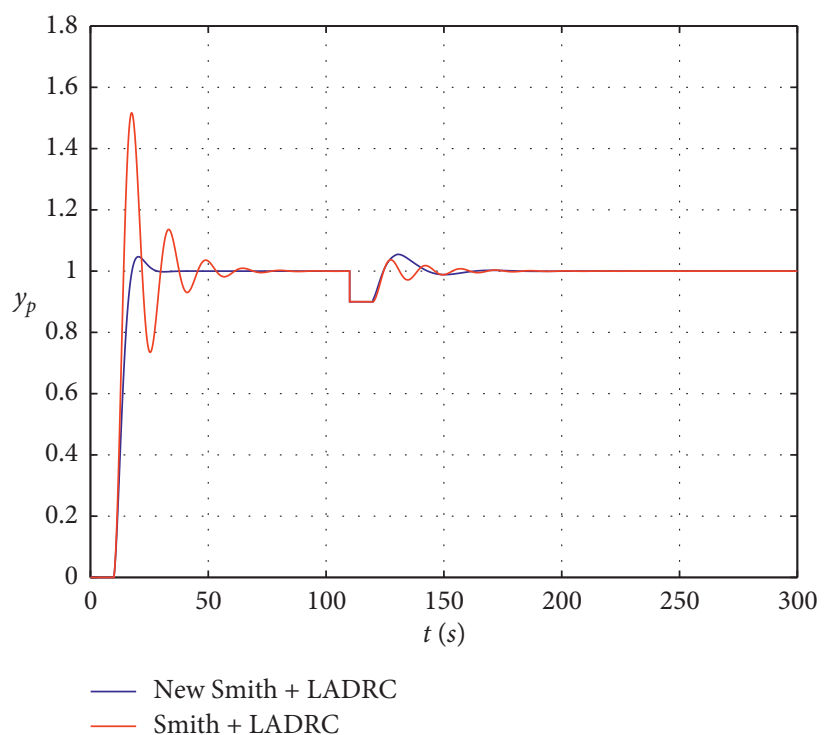

Figure 12: Response curve with $10 \%$ gain reduction.

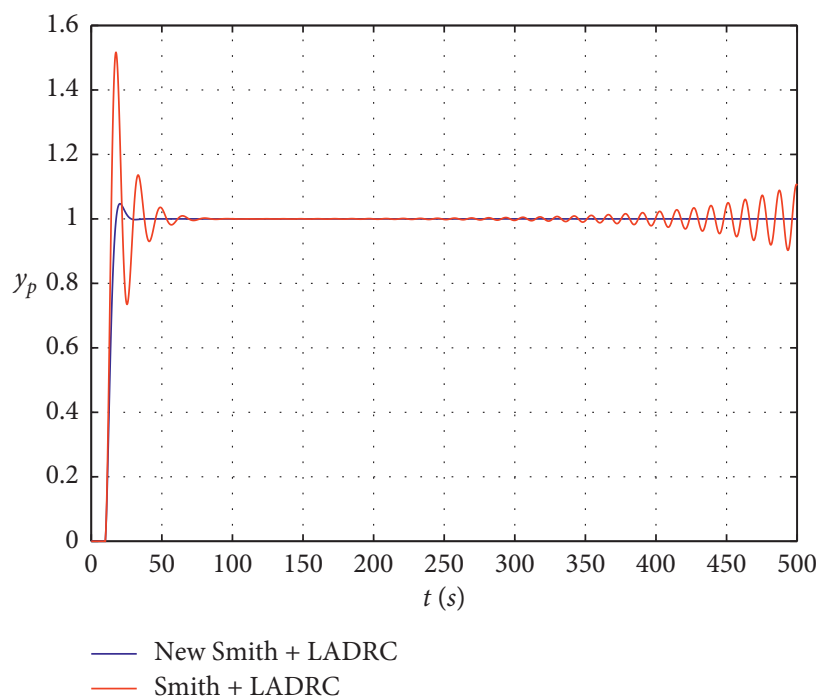

FIGURE 13: Response curve of object $\tau_{p}$ increased by $20 \%$. 


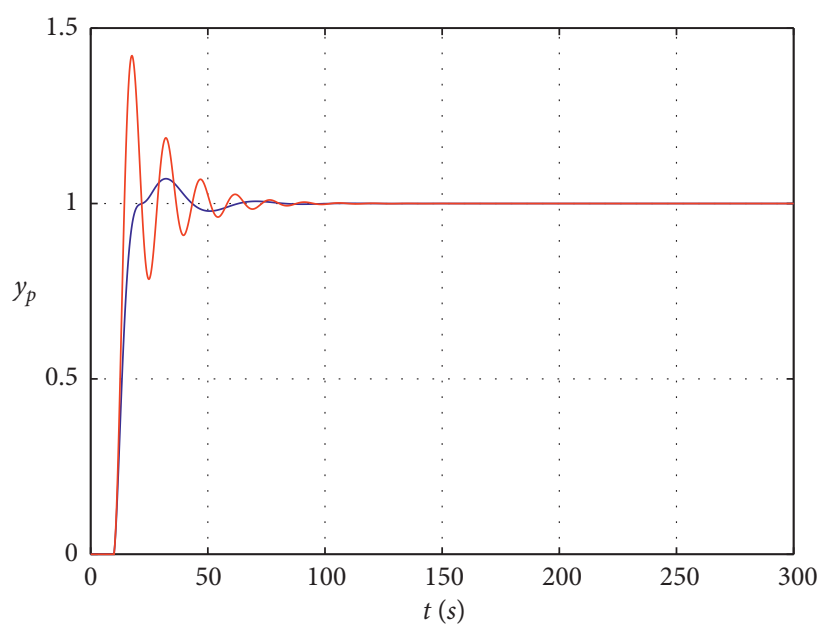

- New Smith + LADRC Smith + LADRC

FIGURE 14: Response curve of the object $T_{p}$ increased by $10 \%$.

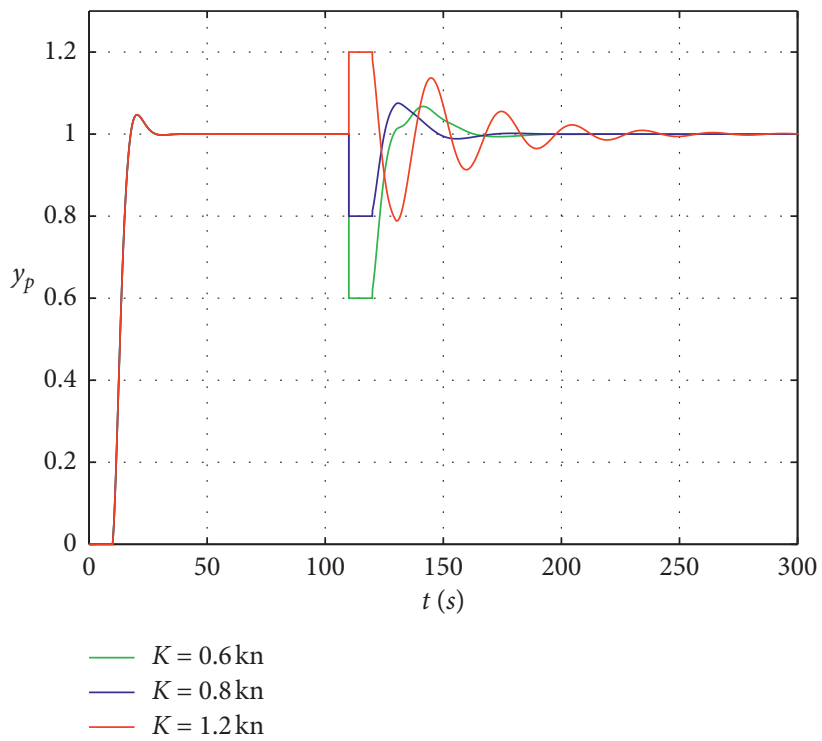

FIgURE 15: Response curve of $y_{p}$ for different $K$.

rise time decreases, and adjustment time becomes longer as well as the steady-state value becoming larger. When $K<K_{n}$, there is no overshoot, rise time increases, and the steadystate value is small.

\section{Simulations and Discusses}

In simulations, the nominal model of a large time-delay system is $G_{b}=G_{n} e^{-\tau_{n} s}=(2 /(10 s+1)) e^{-10 s}$, and parameters of the controller of this nominal model are $k_{p}=20, \omega_{0}=5, b_{0}=20$, $K_{A}=2, K_{d}=0.2, K_{c}=0.5, r(t)=1$. Figure 11 shows the response curve of square wave disturbance with amplitude 1 , frequency of $0.5 \mathrm{rad} / \mathrm{sec}$, and $t=100 \mathrm{~s}$ by using ordinary Smith predictor + LADRC strategy and our proposed strategy (new Smith predictor + LADRC strategy). Figure 12 shows the response curve of the sudden reduction of object gain by $10 \%$ when $t=100 \mathrm{~s}$. Figure 13 shows the response curve of object $\tau_{p}$ increased by $20 \%$. Figure 14 shows the response curve of the object $T_{p}$ increased by $10 \%$. Figure 15 shows the response curve when the object gain changes to $0.6 k_{n}, 0.8 k_{n}$, and $1.2 k_{n}$ separately. Figure 16 shows the response curve of object $\tau_{p}=6$, 12 , and 13, respectively. Figure 17 shows the response curve of $T_{p}=0.8 T_{n}, 1.3 T_{n}$, and $1.5 T_{n}$.

It can be seen from Figures 11-14 that the proposed control strategy has better performance than the ordinary Smith ADRC in suppressing external and internal disturbances. The closed loop is adopted to suppress the decrease of output amplitude that is caused by the introduction of $K_{A}$. It also can be seen from Figures 15-17 that the new Smith + LADRC has better robustness. 


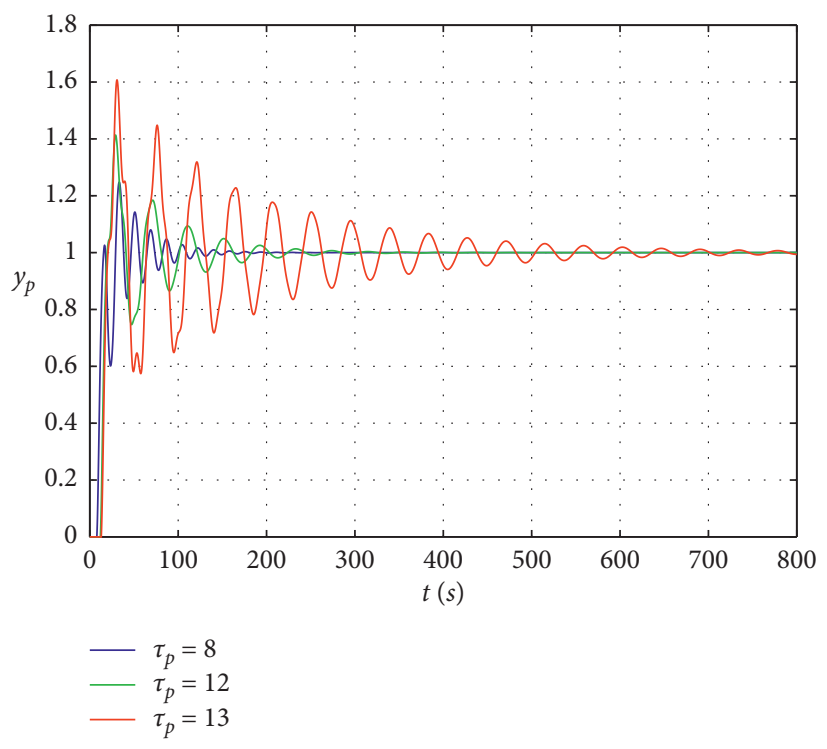

FIGURE 16: Response curve of $y_{p}$ for different $\tau_{p}$.

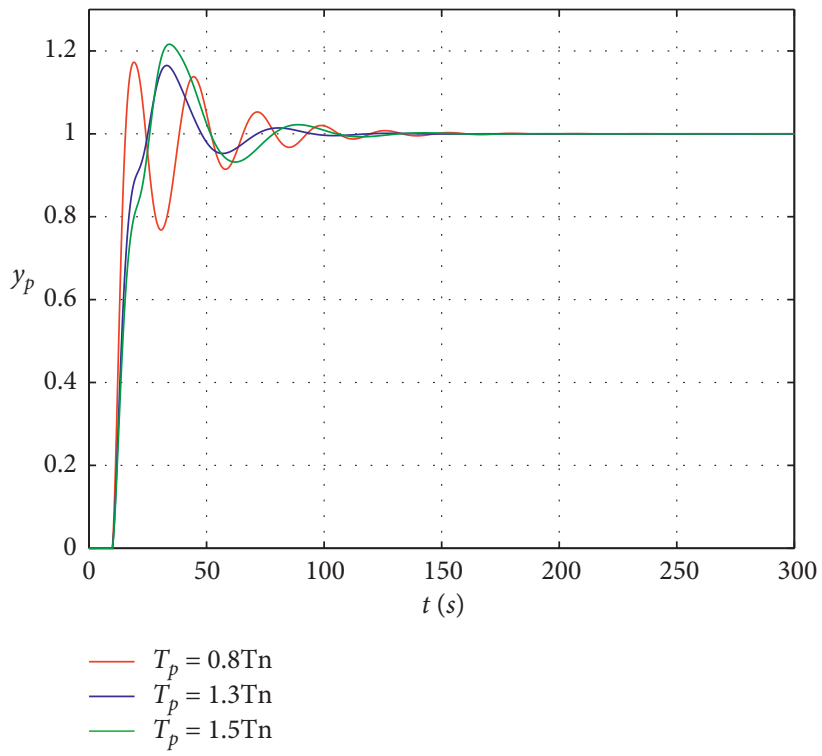

Figure 17: Response curve of $y_{p}$ for different $T_{p}$.

In order to verify the effect of the proposed algorithm, $G_{1}(s)=(0.85 /(1200 s+1)) e^{-1800 s}$ in literature $[1,23]$ is selected as the controlled object. The time-delay parameter $\tau$ is larger than the inertia parameter $T$, which increases the difficulty of control. The controller parameters are selected as $K_{A}=0.01, K_{d}=2, K_{c}=0.2$, respectively. $k_{p}, b_{0}$ are the same as those in literature [1]. The input signal $r(t)=1$, and disturbance signal $r(t-10000)=0.2$. The simulation results are compared with literature [1] in tracking performance and anti-interference performance (literature [1] did not do simulation experiments for each parameter change). Comparison of system response curves can be obtained in Figure 18.
It can be seen from Figure 19 that the control strategy proposed in this paper is superior to the method used in literature [1] in response speed and disturbance rejection.

To further verify the adaptability and robustness of the proposed algorithm, the time-delay system with unstable poles is selected, and its transfer function is $G_{2}=(2 /(10 s-1)) e^{-10 s}$. For this system, the parameters of the controller are selected as $k_{p}=20, \omega_{0}=5, b_{0}=20, K_{A}=3, K_{d}=3, K_{c}=0.53$. Simulation experiments are conducted under various conditions when input signal $r(t)=1$. Figure 20 shows the response curves of the new Smith + LADRC, and Figure 18(b) shows the response curves of the conventional Smith + LADRC. Figure 21 shows the response curve of $T$ change. Figure 22 shows the 


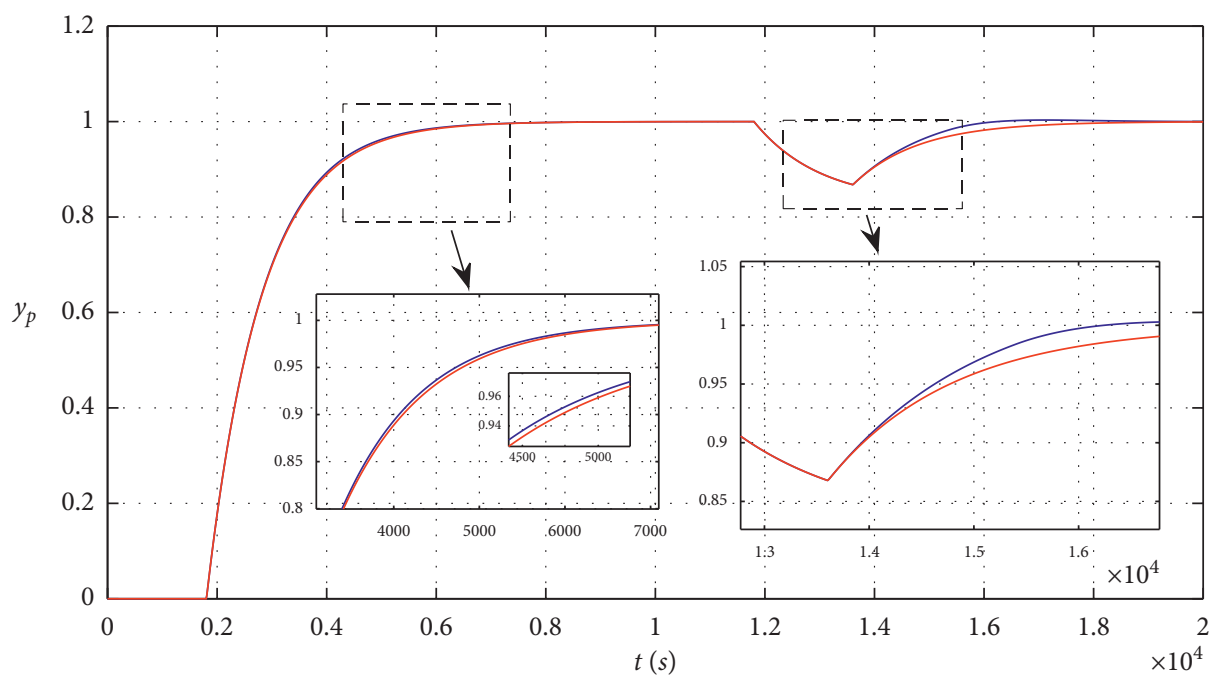

— New Smith + LADRC

- Literature (1)

FIgURE 18: Response curves of the system when the controlled object is $G_{1}(s)$.

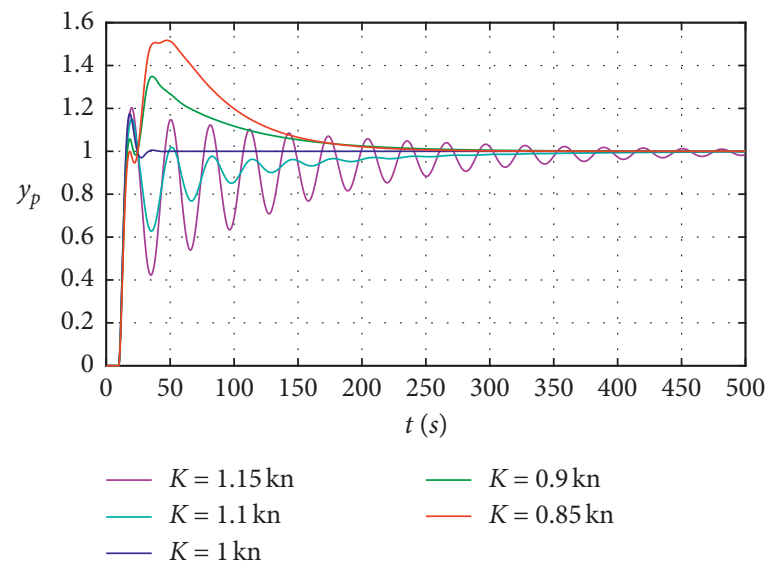

Figure 19: Response curve of $K$ change.

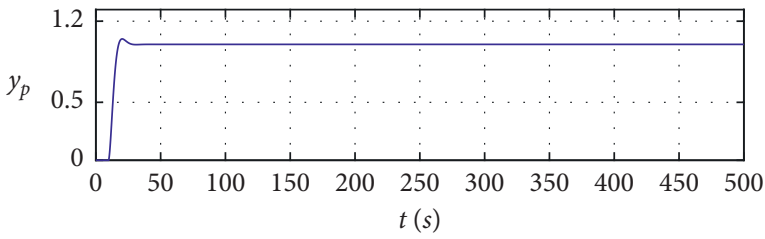

(a)

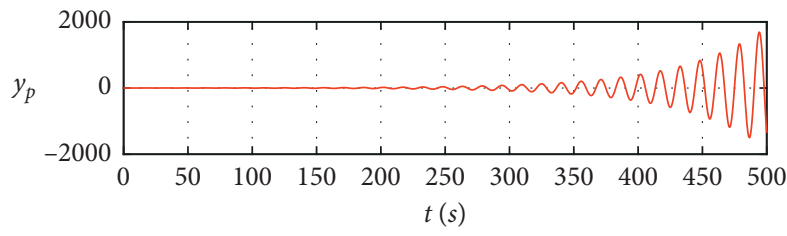

(b)

FIGURE 20: Response curves of the system when the controlled object is $G_{2}(s)$. (a) New smith + LADRC. (b) Conventional smith + LADRC.

response curve of $\tau$ change. Figure 19 shows the response curve of $K$ change.

It can be seen from Figures 19 and 20 that for the timedelay system with unstable poles, the conventional
Smith + LADRC is difficult to achieve stable control, while the new Smith + LADRC proposed in this paper not only can achieve stable control but also has good parameter robustness. 


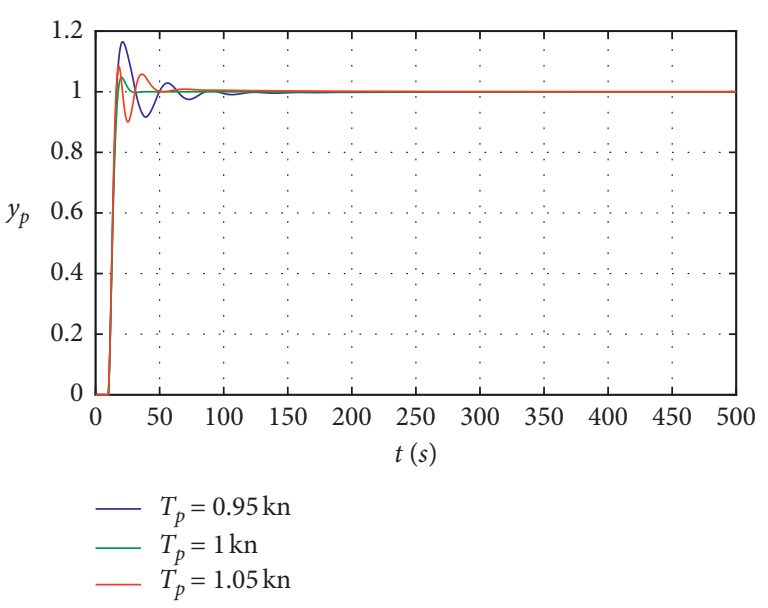

FIgURE 21: Response curve of $T$ change.

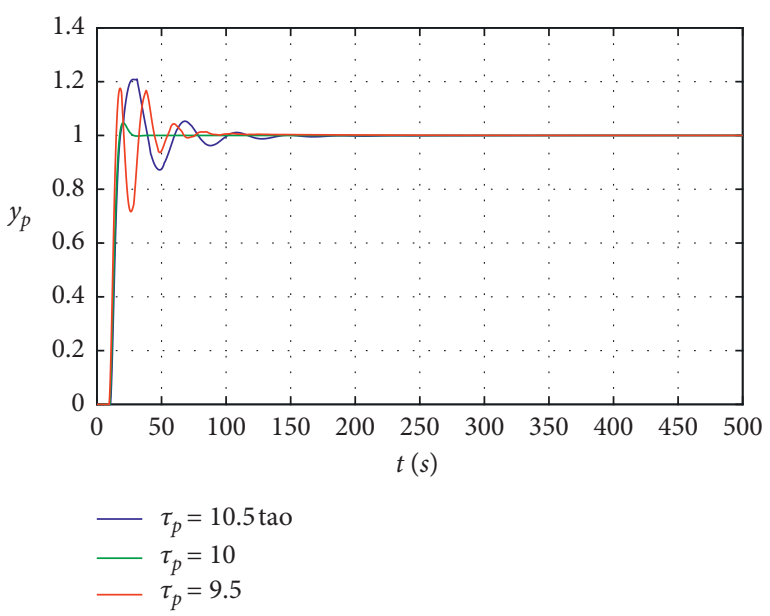

Figure 22: Response curve of $\tau$ change.

\section{Conclusions}

A new Smith + LADRC strategy is proposed to the firstorder large time-delay inertia system. The mechanism of the new Smith predictor and the relationship between parameters and stability of the proposed control strategy are verified via conducting a simulation. Conclusions are as follows:

(1) The performance of the proposed new Smith predictor is not limited by the need to be consistent with the interplant model. It can also be applied to nonminimum phase delay systems.

(2) The robustness of the proposed new Smith predictor is proved by theoretical derivation and numerical simulation. The relationship between parameters and performance indexes is summarized, which can provide guidance for parameters' tuning.

(3) The proposed new Smith predictor + LADRC strategy solves the problem that the input signal of the extended state observer is not synchronized when the LADRC is applied to the time-delay system and has good robustness to parameter disturbance and external disturbance. This strategy has universal applicability for the control problems of time-delay systems.

\section{Data Availability}

The data used to support the findings of this study are available from the corresponding author upon request.

\section{Conflicts of Interest}

The authors declare that there are no conflicts of interest regarding the publication of this paper.

\section{Acknowledgments}

This work was supported by Talent Development Fund Projects of Jilin Province (Grant 201728) and the Science and Technology Research Project of the Education Department of Jilin Province (Grants JJKH20210042KJ and 2015148).

\section{References}

[1] Y. S. Wang, Z. Q. Cheng, M. W. Sun et al., "Robust stability of reduced-order linear adrc for first-order plants with large time-delay," Journal of University of Science and Technology of China, vol. 49, no. 1, pp. 55-62, 2019.

[2] M. E. Koksal, "Commutativity of systems with their feedback conjugates," Transactions of the Institute of Measurement and Control, vol. 41, no. 3, pp. 696-700, 2019.

[3] Z. H. Jiang, X. M. Li, and W. H. Gui, "All parameters adaptive predictive control strategy for long time-delay system," Journal of Central South University (Science and Technology), vol. 43, no. 1, pp. 195-201, 2012.

[4] X. Y. Lou, Z. H. Zhu, and X. P. Guan, "Adaptive sliding mode control for uncertain nonlinear time-delay systems," CAAI Transactions on Intelligent Systems, vol. 5, no. 4, pp. 332-335, 2010.

[5] N. Dong, J. F. Chang, X. S. Han, and A. Wu, "Model-free control method and its application for large time-delay systems," Journal of Harbin Engineering University, vol. 39, no. 12, pp. 1987-1993, 2018.

[6] Q. Zhang, S. X. Feng, and W. Q. Yue, "Control strategy of large volume temperature simulating system based on neural networks," Control Theory \& Applications, vol. 23, no. 3, pp. 430-432, 2006.

[7] X. U. Shi, "An implementation method on intelligent compensated predictive control for delayed systems," Acta Simulata Systematica Sinica, vol. 15, no. 11, pp. 1642-1645, 2003.

[8] Q. J. Li, Q. Huang, and J. Li Jin, "Application of smith predicted adaptive fuzzy-PID controller in time-delay system," Ordnance Industry Automation, vol. 30, no. 12, pp. 49-52, 2011.

[9] H. Yang, M. Yang, and F. Wu, "PI and multi-model dynamic matrix control of reactor-heat exchanger networks," Journal of Chemical Industry and Engineering (China), vol. 56, no. 6, pp. 1470-1478, 2008.

[10] S. Tufenkci, B. Senol, B. B. Alagoz, and R. Matušů, "Disturbance rejection FOPID controller design in v-domain," Journal of Advanced Research, vol. 25, no. 9, pp. 171-180, 2020.

[11] L. J. Wang, Q. Li, C. N. Tong, and Y. Yin, "Overview of active disturbance rejection control for systems with time-delay," 
Control Theory \& Applications, vol. 30, no. 12, pp. 1521-1533, 2013.

[12] S. H. Wang, G. F. Sun, and S. G. Liu, "Active disturbance rejection sliding mode control for time-delay systems," Journal of System Simulation, vol. 31, no. 1, pp. 102-109, 2019.

[13] Y. Cheng, Z. Q. Chen, M. W. Sun, and Q. Sun, "Multivariable inverted decoupling active disturbance rejection control and its application to a distillation column process," Acta Automatica Sinica, vol. 43, no. 6, pp. 1080-1088, 2017.

[14] L. J. Wang, C. N. Tong, Q. Li, and Y. X. Yin, "Practical active disturbance rejection solution for monitoring automatic gauge control system with large time-delay," Control Theory \& Applications, vol. 29, no. 3, pp. 368-374, 2012.

[15] Z. Q. Gao, "Scaling and bandwidth-parameterization based controller tuning," in Proceedings of the 2003 American Control Conference, pp. 4989-4996, Denver, CO, USA, June 2003.

[16] Z. Q. Chen, M. W. Sun, and R. G. Yang, "On the stability of linear active disturbance rejection control," Acta Automatica Sinica, vol. 39, no. 5, pp. 574-580, 2013.

[17] C. B. Wang, Y. Wang, and Q. Liang, "Reduced order active disturbance rejection control for time-delay systems," Control Engineering of China, vol. 23, no. 10, pp. 11602-11606, 2016.

[18] D. Z. Li, W. L. Yu, and Q. B. Jin, "Stability region analysis of linear active disturbance rejection controllers for first order system with time delay," Control Theory \& Applications, vol. 34, no. 9, pp. 1244-1249, 2017.

[19] Z. Q. Cheng, X. Wu, M. W. Sun et al., "Performance analysis of active disturbance rejection generalized predictive control on time-delay systems," Journal of Central South University (Science and Technology), vol. 49, no. 8, pp. 1961-1970, 2018.

[20] Y. S. Wang, Z. Q. Chen, M. W. Sun et al., "Smith prediction and active disturbance rejection control for first order inertial systems with long time-delay," CAAI Transactions on Intelligent Systems, vol. 13, no. 4, pp. 500-508, 2018.

[21] C. Q. Chen, Y. G. Wang, M. W. Sun et al., "A new type of active disturbance rejection control with smith and its robustness for non-minimum phase time-delay systems," Journal of Dalian University of Technology, vol. 59, no. 1, pp. 88-96, 2019.

[22] Q. Zheng and Z. Gao, "Predictive active disturbance rejection control for processes with time delay," ISA Transactions, vol. 53, no. 4, pp. 873-881, 2014.

[23] D. C. Tang, Z. Q. Gao, and X. H. Zhang, "Design of predictive active disturbance rejection controller for turbidity," Control Theory \& Applications, vol. 34, no. 1, pp. 101-108, 2017. 